\title{
Characterisation of crack tip fields under non-uniform fatigue loading
}

\author{
D. Nowell, M.E. Kartal \\ University of Oxford, Department of Engineering Science, Parks Road, Oxford, OX1 3PJ, UK.
}

P.F.P. de Matos

U3Is, Unidade de Investigação e Internacionalização do ISVOUGA, Santa Maria da Feira, Portugal.

\begin{abstract}
The paper analyses previously reported work, which uses digital image correlation to measure fatigue crack closure. As well as determining crack opening loads, the information on crack shape may be used to estimate the stress intensity factor, as well as other parameters in more complex models of crack tip fields. A number of specimens were subjected to single overload cycles, which produced a significant retardation in crack growth rate. The method previously applied to the analysis of constant amplitude loading is here used to analyse the single overload case. The stress intensity factor history is found to be very different in the two cases and the consequences of this observation for analysis of fatigue crack propagation are discussed.
\end{abstract}

KEYWORDS. Digital image correlation; Displacement measurement; Crack-tip fields; Elastic-plastic fatigue crack.

\section{INTRODUCTION}

$\mathrm{T}$ he study of crack tip stress, strain, and displacement fields has a long history. One hundred years ago, Inglis recognized that a sharp elastic crack would exhibit a stress (and strain) singularity [1]. Twelve years later Westergaard [2] produced a stress function solution for a crack in a biaxial stress field which formed the basis for the later concept of the stress intensity factor. An excellent compilation of seminal papers in the field has been provided by Sanford [3,4]. The stress intensity factor has proved to be an extremely useful concept for the assessment of cracks and it has been widely used in industry. Paul Paris' extension of the concept to its use in fatigue crack propagation [5] is particularly worthy of mention. However, it is clear that the stress intensity approach has many limitations. In particular, all fatigue cracks must exhibit some irreversibility at or close to the crack tip, otherwise they would not propagate. The stress intensity approach attempts to characterize this by a similitude argument, which depends on the assumption of small scale yielding (i.e. that the process zone of the crack tip is contained within a wider 'K-dominant' region). Use of the stress intensity approach has particular limitations for the case of non-uniform loading, since load history effects are difficult to capture. Hence, there has been increasing interest in improved characterization of crack tip fields. In parallel, new experimental techniques, such as digital image or volume correlation (DIC/DVC); X-ray tomography; and large scale synchrotron facilities have provide tools which allow various models of crack tip behavior to be tested.

A significant number of papers were presented at the First I.J. Fatigue \& FFEMS Joint Workshop on the Characterisation of Crack Tip Stress Fields held in Forni di Sopra in 2011. This resulted in the publication of two special issues of the journals $[6,7]$, but the workshop showcased only a small proportion of the work in the field. On the experimental side, other recent papers of note include tomographic studies of cracked specimens under live loading [8] and in-situ SEM 
studies of deformations in the crack tip region. The latter work has led to a suggested life prediction approach which obviates the need to characterize the number of cycles experienced by the crack [9]. Despite the existence of rainflow and similar approaches, cycle counting can be a difficulty in non-uniform loading situations. The experimental work reported in the current paper springs from an investigation of crack closure originally carried out by de Matos [10]. However, the data was later re-processed to compare with a two-parameter crack tip field model proposed by Pommier and co-workers [11]. This work was reported at the Forni di Sopra workshop [12] and in the subsequent special issue [13]. However, the majority of the work reported was concerned with constant amplitude loading. A few experiments on simple overload cycles were carried out and the results of one of these experiments were presented in [13]. The displacement fields were converted into the two parameter description $(K, \rho)$ and provided an interesting contrast to the results obtained from constant amplitude loading. The current paper therefore sets out to explore these results in more detail and to analyse a wider range of variable amplitude experiments.

\section{EXPERIMENTAL WORK}

he fatigue experiments carried out by de Matos have been reported elsewhere, but a brief summary is given here for clarity. Fig. 1 shows the main experimental set-up, which employs a CT specimen manufactured from 6082 T6 aluminium alloy. The specimen is loaded in fatigue and the area along the crack flanks is monitored using a Questar long range microscope and a digital camera (a low-cost USB webcam). A second camera is used to measure the crack length on the opposite side of the specimen, but this does not provide sufficient resolution for displacement measurement. The use of the microscope means that only a small area (approx. $600 \times 400 \mu \mathrm{m}$ ) close to the crack tip is imaged, but this permits high resolution and high accuracy evaluation of displacements. It should be noted that, because the primary focus of the investigation was crack closure, most of the image is along the crack wake, and there is very little information collected from the region ahead of the crack tip.

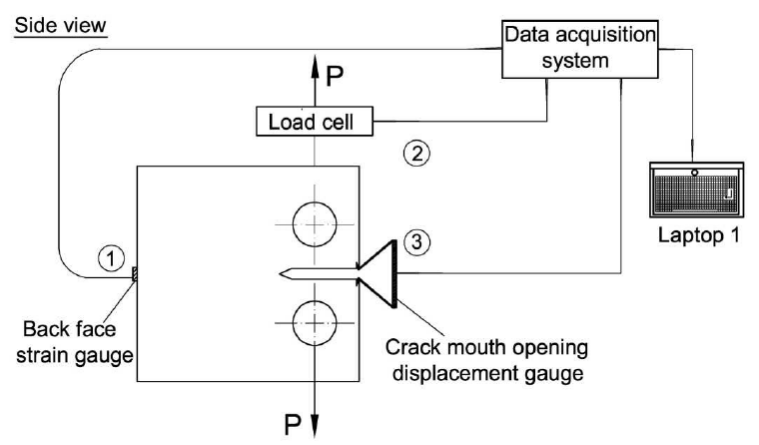

$\underline{\text { Top view }}$

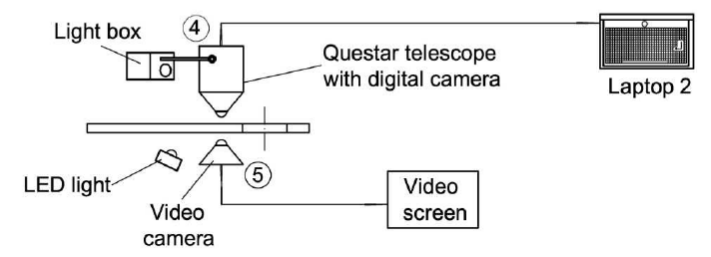

Figure 1: Experimental configuration: side and top views.

Images were collected during fatigue loading at 30fps, whilst the specimen was loaded in a servo-hydraulic test machine at a frequency of $0.25 \mathrm{~Hz}$. A set of 360 images was collected over four loading cycles at a number of discrete points during crack propagation. The loading frequency was increased between these measurement steps in order to propagate the crack in a reasonable time interval. Each set of images was analysed using a public domain Matlab script [14]. Rather than analyse a full displacement field, the relative displacement of five pairs of points along the crack flank was determined (Fig. 2). This information proved useful in investigating crack closure directly, but may also be employed to determine crack tip loading history. The elastic model for a sharp crack predicts that the displacement along the crack faces is given by: 


$$
u_{i}(r)= \pm \frac{4 K_{I}}{E} \sqrt{\frac{r}{2 \pi}}
$$

so that a plot of $\log$ (relative displacement) against $\log (\mathrm{r})$ may be used to estimate the stress intensity factor at any particular time during the crack loading history.

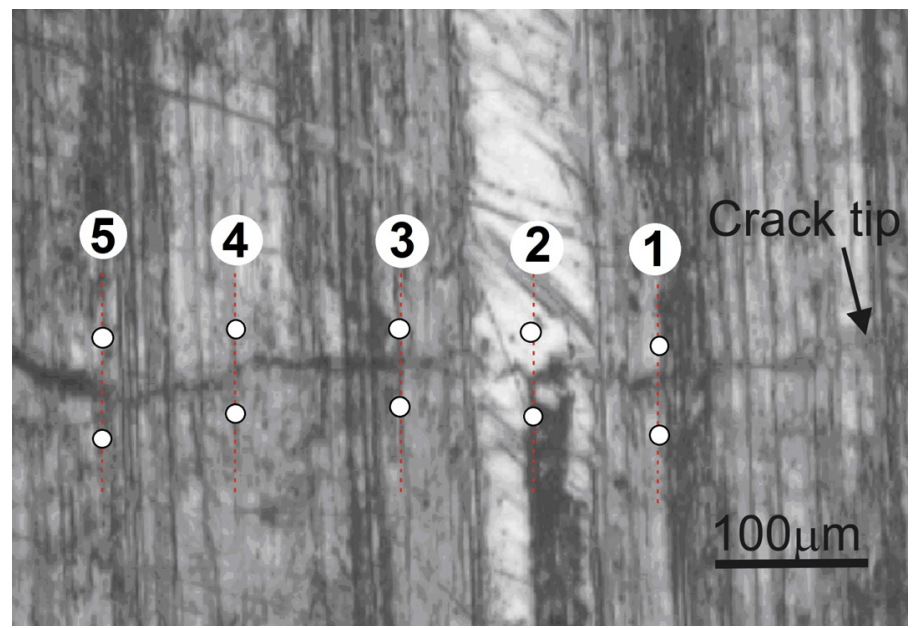

Figure 2: Typical image showing the crack tip, together with the five pairs of measurement points.

\section{RESULTS}

$\mathrm{W}$

hilst the main experimental programme concerned the investigation of behaviour under constant amplitude loading, a small number of specimens were subjected to a single overload in an otherwise constant amplitude load history. Analysis of this situation represents a useful first step towards a full theory which can be applied in any general loading case. Fig. 3a shows the schematic loading history, whereas Fig. 3b shows crack growth rate data (plotted against nominal $\Delta \mathrm{K}$ ) for a number of $3 \mathrm{~mm}$ thick specimens, including two (CTF6 and CTF8) which were subjected to an overload. The resulting retardation in crack growth rate can clearly be seen.

(a)

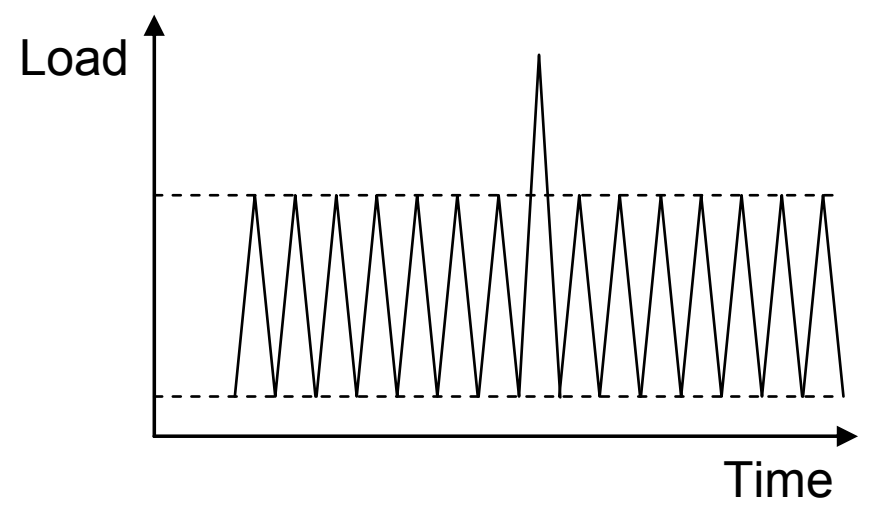

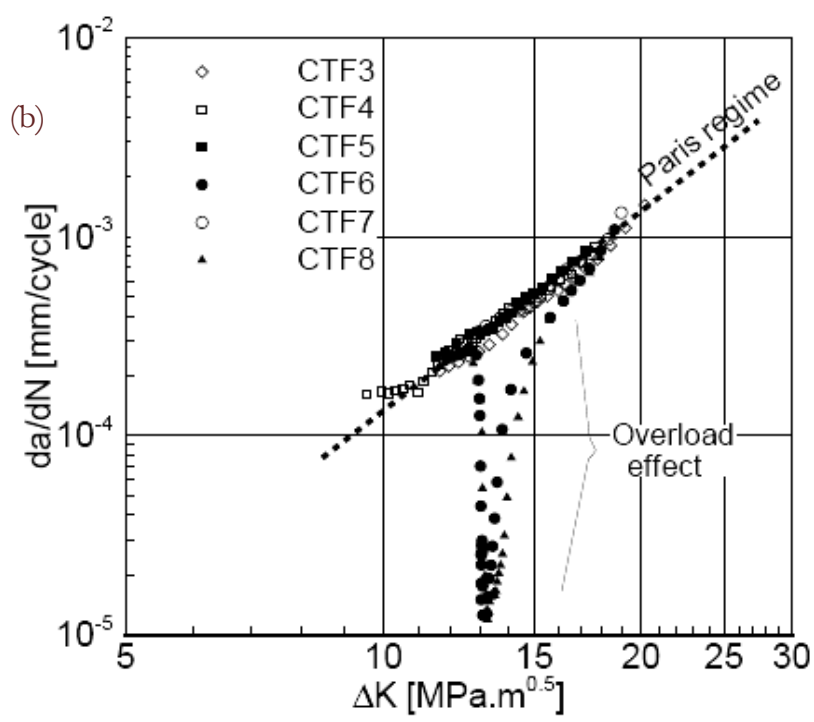

Figure 3: (a) Schematic load history for single overload specimen; (b) Variation of crack growth rate with nominal elastic $\Delta \mathrm{K}$ for $3 \mathrm{~mm}$ thick specimens, including two with single overloads. 
The experimental data is processed as described above and used to determine the stress intensity factor history for the crack. In the case of specimen CTF6, the variation of $\mathrm{K}$ with load, just before the overload, is shown in Fig. 4(a). It can be seen that the behaviour is quite similar to that predicted by an elastic analysis of the specimen geometry. However, there is an offset between the theoretical and experimental lines, caused by crack closure. Alternatively, this phenomenon may be thought of as a residual (negative) $\mathrm{K}$ caused by the residual stress (and displacement) field present. The two lines have similar slope since, once the crack is fully open at a load of approximately $0.5 \mathrm{kN}$, the behaviour is predominantly elastic, with only a small zone of cyclic plasticity close to the crack tip.

(a)

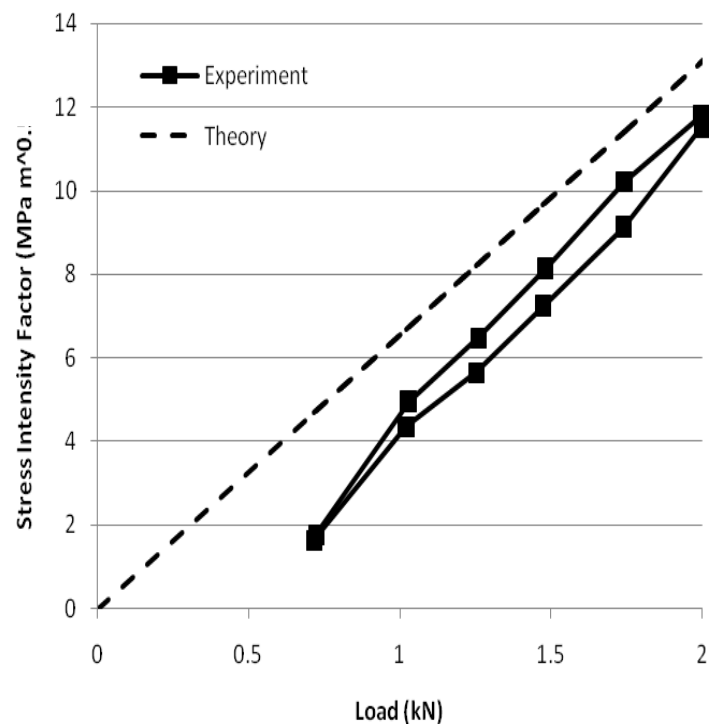

(b)

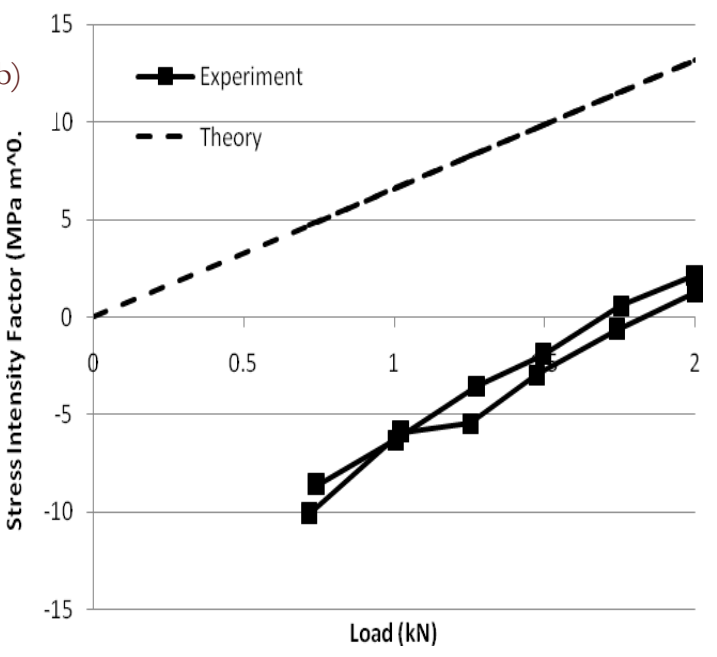

Figure 4: Variation of measured stress intensity factor with load for specimen CTF6 (a) Before the overload and (b) Immediately after the overload.

Fig. 4b shows equivalent data just after the overload. A cursory inspection suggests that the behaviour appears as expected. The slope of the curve remains approximately parallel to the elastic line, and the offset has increased (perhaps as a result of the increased magnitude of residual stress resulting from the overload). However, closer inspection reveals some unexpected features. The analysis yields negative stress intensity factors, which we would not expect to be physically admissible on their own. This must mean that the relative displacement between the crack flanks is varying as $\alpha \sqrt{r}$, where $\alpha$ is a positive constant. Further, this would imply that the stress field at the tip of the crack is compressive and reasonably well described by the singular Westergaard solution. One possible explanation for these observations is that the crack is being held open by the additional crack opening displacement caused by the overload. Investigation of the crack profile broadly confirms this view. As the crack propagates after the overload, the behaviour gradually returns to that which exists previously. Fig. 5 shows a similar plot of load against $\mathrm{K}$ taken 23,500 cycles after the overload. The negative offset is substantially reduced, although negative stress intensity factor values still exist. Moreover it is apparent that the variation is no longer linear over the range of load plotted. The deviation from linearity at lower load values is almost certainly caused by the onset of closure, which was absent immediately after the overload.

The discussion above suggests that a single parameter, $K$, may be insufficient to fully characterize the crack tip environment, particularly under non-uniform loading conditions. An improvement can almost certainly be obtained by explicitly acknowledging the role of crack tip plasticity. A simple model has been proposed by Pommier and Hamam [11] which relies on partitioning the displacement field into elastic and plastic components. The elastic component, $u_{e l}$, is the usual field associated with $K$, whereas the plastic field, $u_{p}$, is associated with crack tip opening. To a first approximation it may be taken as that given by a unit dislocation located at the crack tip. Hence

$$
u=K_{I} u_{e l}+\rho u_{p l}
$$

As far as the relative displacements between the crack faces are concerned, this leads to

$$
u_{y}(r)=\frac{8 K_{I}}{E} \sqrt{\frac{r}{2 \pi}}+\rho
$$


Hence, a plot of $u_{y}$ against $\sqrt{ }_{r}$ should give a straight line with a gradient of $8 \mathrm{~K}_{\mathrm{I}} /(\mathrm{E} \sqrt{ }(2 \pi))$ and an intercept of $\rho$, and values of $K$ and $\rho$ may be obtained from the experimental results in a similar manner to the purely elastic case. Fig. 6 shows the variation of $K$ with $\rho$ for the cycle soon after the overload (i.e. corresponding to the elastic analysis shown in Fig. 5b). This shows evidence of the hysteresis in of $K / \rho$ space, which Pommier and Hamam suggest is associated with fatigue crack propagation. According to their hypothesis, absence of a cyclic variation in $\rho$ corresponds to loading beneath the threshold value of $\Delta K$.

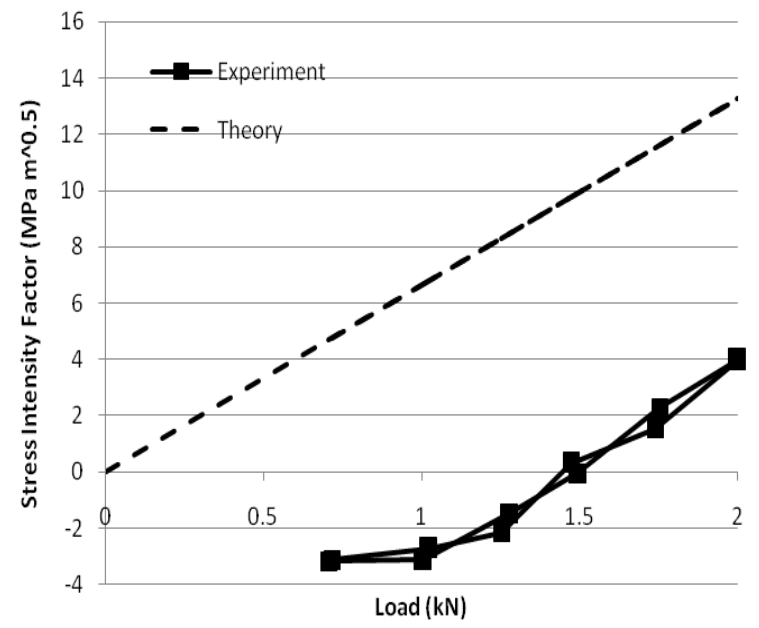

Figure 5: Variation of measured stress intensity factor with load for specimen CTF6, 23,500 cycles after the overload.

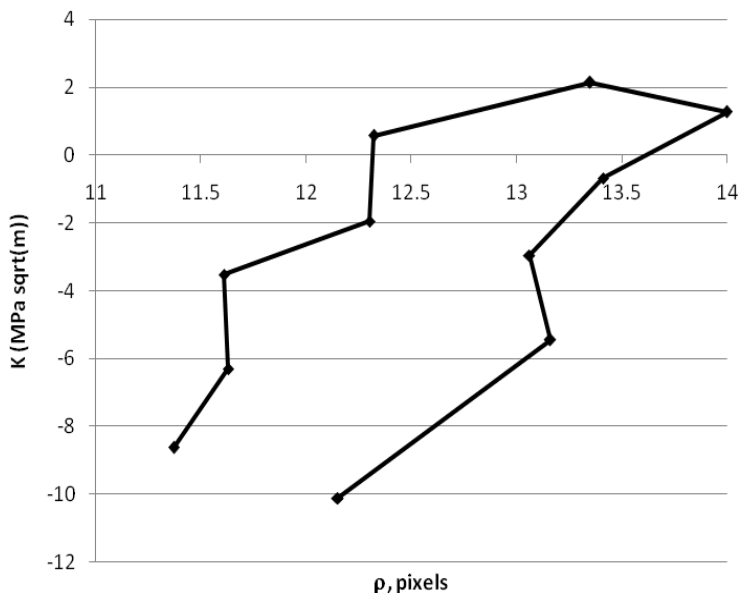

Figure 6: Variation of $K$ with $\rho$ when the elastic/plastic approach is used to analyse a cycle after the overload.

\section{CONCLUSIONS}

he results presented here have demonstrated that displacement information collected in the crack wake can be analysed using digital image correlation in order to give reliable values of the stress intensity factor. For the case of constant amplitude loading, the results are broadly as expected and show a linear relationship of $K$ with load, suggesting that the behaviour is predominantly elastic. There is, however, an offset to the experimental curve, compared with the linear elastic model, which can be attributed to the existence of crack closure. The situation when a non-uniform load history is examined appears more complex. Here, a simple case of a single overload in an otherwise constant amplitude loading has been examined. Even this relatively simple load history gives rise to some surprising results. For some time after the overload, negative stress intensity factors are measured when using a purely elastic model. Normally it is only possible to obtain positive values, as the crack closes and allows transmission of compressive forces across the 
crack faces. The values measured here can only be possible when a significant crack tip opening displacement is also present. This suggests that a more sophisticated description of crack tip fields is necessary, perhaps including elastic and plastic terms as suggested by Pommier and Hammam [11]. Future work will include the comparison of the measured results with strip yield and finite element models. Further experiments are also planned, which will look at the region ahead of the crack tip as well as along the crack flanks. This should allow correlation of the results obtained in the crack wake with conditions in and around the process zone itself.

\section{ACKNOWLEDGEMENT}

$\mathrm{P}$

.F.P. de Matos would like to gratefully acknowledge the support of the Portuguese Fundação para a Ciência e a Tecnologia (FCT) for providing a D.Phil. scholarship (reference SFRH/BD/12989/2003, financed by POSI).

\section{REFERENCES}

[1] Inglis, C.E., Stresses in a plate due to the presence of cracks and sharp corners, Trans of the Institution of Naval Architects, 55 (1913) 219-230.

[2] Westergaard, H.M., Bearing pressures and cracks, Jnl Appl. Mech., 6 (1939) 49-53.

[3] Sanford, R.J., Selected Papers on Foundations of Linear Elastic Fracture Mechanics, SEM/SPIE, USA (1997).

[4] Sanford, R.J., Selected Papers on Crack Tip Stress Fields, SEM/SPIE, USA (1997).

[5] Paris, P., Erdogan, F., A critical analysis of crack propagation laws, Jnl Basic Engineering, 85 (1963) 528-534.

[6] James, M.N., Yates, J.R., Susmel, L., Iacoviello, F., Nowell, D., Lazzarin, P., Patterson, E.A., Carpinteri, A., Characterisation of Crack Tip Stress Fields, Proceedings of the 1st Joint International Journal of Fatigue/Fatigue \& Fracture of Engineering Materials \& Structures conference, held in Forni di Sopra, UD, ITaly, 7-9 March, 2011, Int. Jnl Fatigue, 46 (2013) 1-1.

[7] James, M.N., Yates, J.R., Susmel, L., Iacoviello, F., Guest Editorial: Special Issue on Characterisation of Crack Tip Stress Fields, Fatigue Fract. Engng Mater. Struct., 36 (2013) 1-2.

[8] Limodin, N., Rethore, J., Buffiere, J.Y., Influence of closure on the 3D propagation of fatigue cracks in a nodular cast iron investigated by X-ray tomography and 3D volume correlation, Acta Materiala, 58 (2010) 2957-2967.

[9] Zhang, W., Liu, Y., Investigation of incremental fatigue crack growth mechanisms using in situ SEM testing, Int. Jnl Fatigue, 42 (2012) 14-23.

[10] de Matos, P.F.P., Nowell, D., Experimental and numerical investigation of thickness effects in plasticity-induced fatigue crack closure, Int Jnl Fatigue, 31 (2009) 1795-1804.

[11] Pommier, S., Hamam, R., Incremental model for fatigue crack growth based on a displacement partitioning hypothesis of mode I elastic-plastic displacement fields, Fatigue Fract. Engng Mater. Struct., 30 (2006) 582-598.

[12] Nowell, D., Kartal, M.E., de Matos, P.F.P., Measurement and modelling of near-tip displacement fields for fatigue cracks in 6082 T6 aluminium, Proc. First I.J. Fatigue \& FFEMS Joint Workshop, Forni di Sopra, Italy, March 7-9, 2011, Gruppo Italiano Frattura, 2011.

[13] Nowell, D., Kartal, M.E., de Matos, P.F.P., Digital image correlation measurement of near-tip fatigue crack displacement fields: constant amplitude loading and load history effects, Fatigue Fract. Engng Mater. Struct., 36 (2013) 3-13.

[14] Eberl, C. Thompson, R., Gianola, R., Digital image correlation and tracking with Matlab, Matlab Central file exchange (2006) http://www.mathworks.co.uk/matlabcentral/fileexchange/12413-digital-image-correlation-and-tracking. 